\title{
Medical Family Therapy: Opportunity for Workforce Development in Healthcare
}

\author{
Jennifer Hodgson • Angela Lamson • Tai Mendenhall • \\ Russell Crane
}

Published online: 22 May 2012

(C) Springer Science+Business Media, LLC 2012

Health care in the United States is failing; the system as we know it is in financial ruins (e.g., Himmelstein et al. 2009; World Health Organization 2000). As the prevalence of chronic illness and health disparities continues to increase, many healthcare systems maintain that they are operating through a fragmented model of care that is inefficient, expensive, and ripe with opportunities for over-treatment, under-treatment, and misdiagnosis (Dixon and Samarth 2009; Institute of Medicine 2001). Systems that function in "disciplinary silos" result in medical contexts that are void of psychosocial assessments and indicated treatments when patients are faced with symptoms that are perceived solely through a physical health lens. The same occurs in mental health venues wherein medical conditions, providers, and prescriptions are not considered when gathering information about a family's history, setting clinical goals, or planning treatment. A potential resolution to these challenges was put into motion in March 2010 when the Patient Protection and Affordable Care Act (PPACA) was signed into law, providing an opportunity to redesign healthcare delivery.

Given that approximately $70 \%$ of patients who are seen in primary care have a psychosocial issue (Follette and Cummings 1967; Fries et al. 1993; Gatchel and Oordt 2003;

\footnotetext{
J. Hodgson $(\bowtie)$

Departments of Child Development \& Family Relations and Family Medicine, East Carolina University, Greenville, NC 27858, USA

e-mail: hodgsonj@ecu.edu
}

\author{
A. Lamson \\ Department of Child Development \& Family Relations, East Carolina University, \\ Greenville, NC 27858, USA \\ e-mail: lamsona@ecu.edu \\ T. Mendenhall \\ Department of Family Medicine and Community Health, University of Minnesota Medical School, \\ 717 Delaware St. SE, Suite 422, Minneapolis, MN 55414, USA \\ e-mail: mend0009@umn.edu \\ R. Crane \\ School of Family Life, Brigham Young University, 257 Taylor Building, Provo, UT 84602, USA \\ e-mail: russ_crane@byu.edu
}


Kroenke and Mangelsdorf 1989) and that only about $25 \%$ of patients who receive a mental health referral by a medical provider to an off-site location actually attend psychotherapy (Druss et al. 2008), providing care through disciplinary silos is at least inefficient. As care sites are increasingly co-locating and integrating medical and mental health care services, fewer patients and families are potentially left under-treated.

Family therapists, grounded in a systemic and relational lens, are well positioned to be leaders in this evolution across integrated and collaborative care services in primary, secondary, and tertiary care settings. Medical Family Therapy (MedFT), specifically, was originally advanced through a shared vision by Susan McDaniel, Bill Doherty, and Jeri Hepworth in the early 1990s. They recognized that the application of family therapy's systemic thinking offered a biopsychosocial sensitivity to providing patients and families. They saw an opportunity for mental health providers to be trained to intervene in healthcare settings and with traditionally "medical" issues. Since then, researchers have noted the impact of health on families and visa-versa (Burman and Margolin 1992; Fan and Chen in press; Robles and Kiecolt-Glaser 2003; Wickrama et al. 2001). While McDaniel et al. (1992) envisioned MedFT as more of a metaframework rather than as a subdiscipline of family therapy, family therapists have moved forward with initiating training programs, certificates, and degrees in MedFT that provide mental health clinicians with intensive training in family therapy and its application in healthcare systems targeting medical conditions.

In 2007, Linville et al. noted that MedFT needed more research, but more importantly that it lacked a cohesive definition because so many authors had added their own concepts to it since it was first developed by McDaniel et al. (1992). Therefore, in 2010 Tyndall et al. embarked on a study using a Dephi method (Marchais-Roubelat and Roubelat 2011; Rowea and Wright 2011) to find out how experts were defining MedFT. The following is the definition that formally resulted. Medical Family Therapy is:

an approach to healthcare sourced from a BPS-S [biopsychosocial-spiritual] perspective and marriage and family therapy, but also informed by systems theory. The practice of MedFT spans a variety of clinical settings with a strong focus on the relationships of the patient and the collaboration between and among the healthcare providers and the patient. MedFTs are endorsers of patient and family agency and facilitators of healthy workplace dynamics (Tyndall et al. 2010).

This new definition affirmed that many of the concepts highlighted 20 years ago are still critical to the implementation of MedFT today. However, the need for more overt inclusion of spirituality as a dimension of care and collaboration as a vehicle to successful intervention of patient-care and workplace dynamics was strongly punctuated.

This special issue includes a range of articles designed to perturb our field to think about how we can better train and integrate ourselves to be valuable in healthcare settings, research, and policy. As described above, Susan McDaniel, Bill Doherty, and Jeri Hepworth first disseminated their ideas when they published their primer on Medical Family Therapy in 1992. In 2012, they will publish a second edition of this work.

The first article in the current special issue of Contemporary Family Therapy is an interview facilitated by Barry Jacobs with these pioneers regarding their thoughts about what has happened since their first edition was printed and the potential for MedFT in the future.The remainder of the special issue was carefully crafted with respect to Peek's depiction of a "three world view" (Patterson et al. 2002; Peek 2008). Peek, an innovator in behavioral health integration, has challenged those committed to healthcare to think about it from the viewpoints of clinical, operational, and financial perspectives. Healthcare's clinical world is relevant to the models and approaches that providers use to deliver care to patients and families. 
The operational world is related to the workflow, procedural, and structural (re)design elements of healthcare. The financial world is about how healthcare systems sustain themselves economically, and on what we need to change across clinic-, state-, and federal- levels to do so. We have designed this issue to provide information and innovations at each of these levels. Articles at the clinical levels include Lewis et al.'s biospsychorelational overview of military and veteran couples, Forbat et al.'s qualitative investigation regarding clinical support of caregivers at patients' end-of-life, Fitzgerald and Thomas' report regarding working with couples struggling with medical conditions through attachment perspectives and emotionallyfocused couples therapy, and Skorunka et al.'s family-based efforts with patients struggling with psychosomatic disorders. Articles at the operational levels include Fox et al.'s account regarding the opportunities and challenges for family therapists working in primary care and Marlowe et al.'s framework for making such integration work. Articles at the financial levels include Edwards et al.'s primer for Medical Family Therapists in healthcare policy and Crane and Christenson's summary report of family therapy's cost effectiveness. Articles tying all three of these worlds together include Tyndall et al.'s theoretical and empirical review of MedFT, Mendenhall et al.'s call to advance research in our field, and Tyndall et al.'s consideration of competencies core to our work.

In 2010, the American Association for Marriage and Family Therapy formulated a training track as part of its annual conference devoted to workforce development in MedFT. What is needed now is ongoing training across University training sites and at national conferences to help new and practicing clinicians and researchers grow and develop MedFT, so that they are more competitive in the marketplace. Empirical evidence is also needed that addresses the issues of health using a biopsychosocial-spiritual and systemic lens to generate outcomes that are transformative for patients and their families in-context. While Crane and Christenson (in this special issue) have provided us with some studies, we need more research to demonstrate the health benefits for the couple and family when the patient seeks treatment and members of their family/social systems are included as a part of it. Family therapists are the most prepared to research and manage couple and family dynamics and relational issues. Family therapists are also skilled at helping to resolve issues common to workplace dynamics when providers evidence symptoms of conflict, compassion fatigue, and burnout when trying to provide care in a failing healthcare system.

The editors of the special issue wish to thank all of the contributors to this collection of work. We see this as a catalyst for conversation and opportunity to help train our family therapy workforce to successfully function in healthcare settings as clinicians, researchers, and leaders while applying and studying MedFT concepts and methods. This special issue will also assist those in traditional mental health settings by punctuating the need to strengthen collaboration with other health providers and working with patients through a biopsychosocial-spiritual and systemic lens. While the editors endorse the idea of core competencies in behavioral health integration that span across all mental health disciplines, we challenge family therapists to think more broadly about how their unique skills are useful in healthcare settings, research, and opportunities for local, state, or national policy changes.

\section{References}

Burman, B., \& Margolin, G. (1992). Analysis of the association between marital relationships and health problems: An interactional perspective. Psychological Bulletin, 112, 39-63. doi:10.1037/0033-2909.112.1.39.

Dixon, B., \& Samarth, A. (2009). Innovations in using health IT for chronic disease management: Findings from the AHRQ health IT portfolio. AHRQ Publication No. 09-0029-EF. Rockville, MD: Agency for Healthcare Research and Quality. 
Druss, B. G., Rask, K., \& Katon, W. J. (2008). Major depression, depression treatment and quality of primary medical care. General Hospital Psychiatry, 30, 20-25. doi:10.1016/j.genhosppsych.2007.08.015.

Fan, Y., \& Chen, Q. (2012). Family functioning as a mediator between neighborhood conditions and children's health: Evidence from a national survey in the United States. Social Science \& Medicine, 74, 1939-1947.

Follette, W. T., \& Cummings, N. A. (1967). Psychiatric services and medical utilization in a prepaid health plan setting. Medical Care, 5, 25-35.

Fries, J., Koop, C., \& Beadle, C. (1993). Reducing health care costs by reducing the need and demand for medical services. New England Journal of Medicine, 329, 321-325.

Gatchel, R. J., \& Oordt, M. S. (2003). Clinical health psychology and primary care: Practical advice and clinical guidance for successful collaboration. Washington, DC: American Psychological Association. doi:10.1037/10592-000.

Himmelstein, D. U., Thorne, D., Warren, E., \& Woolhandler, S. (2009). Medical bankruptcy in the United States, 2007: Results of a national study. The American Journal of Medicine, 122, 741-746. doi: 10.1016/j.amjmed.2009.04.012.

Institute of Medicine (U.S.) Committee on Quality of Health Care in America. (2001). Crossing the quality chasm: A new health system for the 21st century. Washington, DC: National Academy Press.

Kroenke, K., \& Mangelsdorf, D. (1989). Common symptoms in ambulatory care: Incidence, evaluation, therapy and outcome. American Journal of Medicine, 86, 262-286.

Linville, D., Hertlein, K. M., \& Prouty Lyness, A. M. (2007). Medical family therapy: Reflecting on the necessity of collaborative healthcare research. Families, Systems, and Health, 25, 85-97. doi:10.1037/ 1091-7527.25.1.85.

Marchais-Roubelat, A., \& Roubelat, F. (2011). The Delphi method as a ritual: Inquiring the Delphic Oracle. Technological Forecasting and Social Change, 78, 1491-1499. doi:10.1016/j.techfore.2011.04.012.

McDaniel, S., Hepworth, J., \& Doherty, W. (1992). Medical family therapy: A biopsychosocial approach to families with health problems. New York: BasicBooks/HarperCollins Publishers, Inc.

Patterson, J., Peek, C., Heinrich, R., Bischoff, R., \& Scherger, J. (2002). Mental health professionals in medical settings: A primer. New York: W.W. Norton \& Co.

Peek, C. (2008). Planning care in the clinical, operational, and financial worlds. In R. Kessler (Ed.), Collaborative medicine case studies (pp. 327-340). New York: Springer.

Robles, T., \& Kiecolt-Glaser, J. (2003). The physiology of marriage: Pathways to health. Physiology \& Behavior, 79, 409-416. doi:10.1016/S0031-9384(03)00160-4.

Rowea, G., \& Wright, G. (2011). The Delphi technique: Past, present, and future prospects. Technological Forecasting and Social Change, 78, 1487-1490. doi:10.1016/j.techfore.2011.09.002.

Tyndall, L., Hodgson, J., Lamson, A., Knight, S., \& White, M. (2010). Medical family therapy: Conceptual clarification and consensus for an emerging profession. Unpublished PhD dissertation, East Carolina University, Greenville.

Wickrama, A., Frederick, L., Wallace, L., Peiris, L., Conger, R., \& Elder, G. (2001). Family influence on physical health during the middle years: The case of onset of hypertension. Journal of Marital \& Family Therapy, 63, 527-539. Article Stable http://www.jstor.org/stable/3654611.

World Health Organization [WHO]. (2000). World health report 2000: Improving performance. Geneva, Switzerland: World Health Organization. 